\title{
Educación emprendedora en la universidad: Educando para el futuro
}

\section{Entrepreneurship education in college: Educating for the future}

Guillermo Gutiérrez Montoya*

\begin{abstract}
Resumen
La educación tiene un impacto positivo en el desarrollo del emprendedurismo, principalmente a nivel de las metodologías utilizadas en el aula. Para que la educación despierte el espíritu emprendedor del estudiante, deben romperse antiguos paradigmas y viejos modelos pedagógicos; se debe evolucionar cualitativamente hacia nuevos métodos, en donde el educando sea protagonista y responsable principal de su propio aprendizaje
\end{abstract}

\section{Palabras clave}

Educación emprendedora, características emprendedoras, estilos de aprendizaje, universidad emprendedora.

\section{Abstract}

Education has a positive impact in the development of entrepreneurship, mainly at the methodologies used in classrooms. To make education awakes the interest in the entrepreneur spirit of students, old- fashion pedagogic models must be broken. It is necessary to develop to new methods where pupils have a leading role and be responsible of his own learning.

\section{Keywords}

Entrepreneurship education, entrepreneurial characteristics, learning styles, entrepreneurial university.

Forma sugerida de citar: Gutiérrez, Guillermo. 2011. Educación emprendedora en la universidad. Educando para el futuro. Retos 2. Julio/diciembre. Pp. 49-68.

\footnotetext{
* Doctor en Ciencias Empresariales por la Universidad de Cádiz (España).Email: guillermo@udb.edu.sv, guillermo. udb@gmail.com
} 
"La mayoría de lo que usted escucha hablar sobre emprendedurismo... está equivocado. No es mágico; no es misterioso; y no tiene nada que ver con los genes. Es una disciplina y, como cualquier disciplina, puede aprenderse"

Peter Drucker, 1985

\section{Introducción}

La estampa del emprendedor se remonta a las primeras agrupaciones humanas y, por tanto, a nuestro pasado más recóndito como civilización. Sus características, casi inalterables en el transcurso del tiempo, lo sitúan siempre en una posición relevante y fundamental en el desarrollo y progreso social. No obstante, al referirnos a emprendedores debemos huir de la idea de la existencia de "mentes privilegiadas", como Geneen y Moscow (1984) lo comprobaron, son personas comunes las que se transforman en líderes empresariales. También debo hacer notar que no todo emprendedor es aquella persona que forma empresas, sino que también tenemos los intraemprendedores (Pinchot, 1985), que son quienes trabajan al interior de una empresa y se constituyen como los mejores empleados dentro de la misma, aportando ideas, trabajando proactivamente para la empresa, se desarrollan en la empresa, etcétera. Otra distinción es la del emprendedor social (López, 2005), que desarrolla proyectos que benefician a la sociedad en general, como el caso de San Juan Bosco, visionario persistente.
El presente trabajo investiga el fenómeno del emprendedurismo desde la óptica académica, analizando el impacto que tiene la "educación" en el desarrollo del espíritu emprendedor, como ya lo afirmaba S. Covey (1999), "la verdadera máquina que está conduciendo nuestra economía y sociedad son los emprendedores".

El estudio Global Entrepreneurship Monitor (GEM) (2010) ha demostrado la importancia que el tema del espíritu empresarial ha adquirido, y se ha llegado a convertir en una herramienta para la creación de empleo y generación de riqueza, lo cual demuestra que el crecimiento y el desarrollo económico están ligados al emprendimiento (Acs, Arenius \& Minniti, 2005; José C. Sánchez, 2011).

Revisando la literatura para este artículo, me encontré con la siguiente anécdota que quiero compartirla con ustedes: "se cuenta que en una entrevista exclusiva interrogaron a Bill Gates: ¿Por qué Latinoamérica no ha producido un nuevo Bill Gates?". A lo que él respondió: ¿Usted se habría podido convertir en el innovador tecnológico de más éxito en el mundo, y en uno 
de los hombres más ricos del planeta, si es que hubiera nacido en Paraguay? (Artículo "El Emprendedor Inteligente", de Alberto Conti, 2009). Existen múltiples factores que inciden directa e indirectamente en la aseveración que realiza Bill Gates, pero en este artículo nos vamos a concentrar en uno en particular, la denominada educación emprendedora o educación para emprender.

\section{Conceptos fundamentales del emprendimiento}

Emprendedurismo

La traducción directa de entrepreneurship de la jerga inglesa es ambigua, y se puede traducir como: emprendimiento,[1] empresarialidad, espíritu emprendedor, empresariado. Para efectos de este artículo, su traducción aceptada será emprendedurismo.

El término emprendedurismo se utiliza para referir al sistema de rasgos, valores, cualidades, y actitudes psicológicos personales asociadas fuertemente a una motivación para comenzar una actividad empresarial.[2] La presencia del emprendedurismo no es claramente ninguna garantía que el individuo realmente iniciará una nueva empresa en un cierto punto en su vida. Ni la carencia del emprendedurismo sugiere que nunca se sienta bien como empresario. Tener un espíritu emprendedor significa simplemente que se está predispuesto hacia la actividad emprendedora y que, ceteris paribus, hay una probabilidad substancialmente creciente que algún día elegirá comenzar con una actividad emprendedora y se tendrá éxito (Moen et al., 2004).

Retos 2(1): 2011.

(C) 201 I, Universidad Politécnica Salesiana del Ecuador

\section{Emprendedor}

Según el Diccionario de la Real Academia de la Lengua Española, el emprendedor es aquella persona que "impulsa con resolución acciones dificultosas o azarosas”, además agrega que la palabra emprender (Del lat. in, en, y prende re, coger) en el ámbito de los negocios puede definirse como "acometer y comenzar una obra, un negocio, con empeño, especialmente si encierran dificultad o peligro".

Pinchot (1985) define a un emprendedor como "cualquier soñador que hace" y agrega que "los emprendedores son personas que sueñan y logran hacer realidad lo que imaginan. Son aquellos que pueden tomar una idea y hacerse responsables de innovar y de obtener resultados".

Para Mulcahy (2003) el emprendedor es "una persona que emprende o controla un negocio o empresa, y asume el riesgo de ganancia o pérdida”. El progreso de la humanidad de cavernas a recintos universitarios ha sido explicado de numerosas formas. Pero el centro prácticamente de todas estas teorías ha sido el papel "de agente de cambio", la fuerza que inicia y coloca en práctica el progreso material. Hoy reconocemos que el agente de cambio de la historia humana ha sido y muy probablemente seguirá siendo el emprendedor (Kent, Sexton y Vesper, 1982).

\section{El perfil del emprendedor} y características emprendedoras personales (CEP's)

El perfil emprendedor es descrito por Hisrich (1988) como "las motiva- 
ciones y características personales". De forma que para el establecimiento de un perfil emprendedor, deben tomarse en cuenta las características o rasgos personales que posee un individuo (McClelland, 1985; Pinchot, 1985; Ibrahim y Goodwin, 1986; Ibrahim y Ellis, 1990; Bird, 1989).

A estos rasgos emprendedores se les conoce como CEP's (Características Emprendedoras Personales). Este término fue introducido por la Cooperación Alemana (GTZ) a través del "Programa Emprende", el cual capacitaba con Metodología CEFE a facilitadores para la formación de empresarios emprendedores (véase www.cefe.net).

A través de la revisión teórica, se han logrado detectar las siguientes características que diversos autores le atribuyen al emprendedor de éxito:

\section{El logro de objetivos y metas}

El emprendedor suele aparecer a los ojos de los demás como alguien guiado internamente por un imparable deseo de lograr sus objetivos, como alguien que busca la excelencia. Su fuerte carácter competitivo le lleva a aceptar ciertos riesgos y a perseguir objetivos que le motiven (Kent, Sexton y Vesper, 1982; Montagno y Kuratko, 1986; Begley y Boyd, 1987; Timmons, 1976; Bumm, 1988; Hisrich, 1988; Hatch y Zweig, 2000; Mariotti et al., 2005).

\section{Autoconfianza, perseverancia $y$ dedicación}

La total dedicación a perseguir el éxito posibilita al emprendedor a sobreponerse a los obstáculos derivados de su actividad (Ibrahaim y Soufani, 2002). En muchas ocasiones, la dedicación, la perseverancia y la confianza compensan al emprendedor incluso por sus limitaciones personales (Kuratko y Hodgetts, 1995; Hatch y Zweig, 2000; McGinnis y Frehn, 1987; Kent, Sexton y Vesper, 1982; Montagno y Kuratko, 1986; Begley y Boyd, 1987). En busca del éxito de su proyecto, el emprendedor dedicará interminables jornadas a la empresa, sacrificará su ocio personal, su estancia con la familia, incluso reducirá sus estándares de vida con tal de empujar su proyecto (Hatch y Zweig, 2000; Ibrahim y Ellis, 1990; Bird, 1989; Mariotti et al., 2005). "Energía, determinación y persistencia, se refieren a una reserva de fuerza física y emocional que le permite al individuo innovador que continúe cuando otros podrían rendirse" (McGinnis y Frehn, 1987; Covey, 1999). Estos rasgos Maslow (1943) los cataloga dentro de las necesidades de autoestima y de autorrealización (dar lo que uno es capaz).

\section{Asumen riesgos calculados}

Estudios realizados encontraron que los emprendedores exitosos no buscan deliberadamente situaciones de riesgo elevado, ni se esfuerzan en evitar riesgo en conjunto. Esencialmente están dispuestos a aceptar el riesgo y la incertidumbre inherentes a las nuevas oportunidades Los emprendedores no son jugadores de casino. Cuando éstos deciden perseguir un proyecto empresarial lo hacen calculando al máximo los riesgos (Kuratko 
y Hodgetts, 1989; McClelland, 1987; Bird, 1989; Ibrahim y Ellis, 1990; Mariotti, 2005), analizando el proyecto y su entorno, en definitiva teniendo claro el resultado previsible de su operación (Marinelli, 1998). Además, con su gestión y su esfuerzo personal intentan poner todas las posibles circunstancias a su favor para evitar riesgos innecesarios (Bumm, 1988; Kolchin, 1987; Hatch y Zweig, 2000; Littunen, 2000; Hisrich, 1992; Kent, Sexton y Vesper, 1982; Begley y Boyd, 1987; Montagno y Kuratko, 1986).

Mitton (1989) ha definido al emprendedor como "la persona a quien le gusta tomar riesgos, siempre listo a buscar y manejar situaciones desorganizadas y aceptar riesgos, así como a evitarlos".

Bird (1989) divide el riesgo en cinco tipos, cuatro de los cuales son claramente relevantes a cualquier emprendedor potencial: "riesgo económico, riesgos en las relaciones sociales, riesgo en el desarrollo de la carrera, además el riesgo psicológico y de salud".

\section{Iniciativa}

Al emprendedor le gusta confrontar situaciones en las que se convierte en responsable del éxito o del fracaso de una operación (Mariotti et al., 2005; Kuratko y Hodgetts, 1995). Les atrae tomar la iniciativa para resolver problemas o llenar vacíos de liderazgo. Son personas orientadas a la acción (Kent, Sexton y Vesper, 1982; Begley y Boyd, 1987; Montagno y Kuratko, 1986, Kiggundu, 2002; Shapero, 1975; McGinnis, 1987).

Retos 2(I): 201 I.

(C) 20I I, Universidad Politécnica Salesiana del Ecuador
Son tolerantes a la ambigüedad e incertidumbre

Entendiéndase la ambigüedad e incertidumbre como la capacidad para actuar en un ambiente imprevisible, discordante y ambivalente. Los emprendedores, como todos los empresarios profesionales, se encuentran frente a un continuo cambio, un entorno que aporta ambigüedad e incertidumbre en todos los aspectos de la organización (McClelland, 1987; Bird, 1989; Ibrahim y Ellis, 1990). Los mejores emprendedores navegan entre esa incertidumbre y logran generar ventajas que aprovechan para su organización (Wennekers y Thurik, 1999; Ibrahaim y Soufani, 2002). Para el emprendedor, como para todo aquel relacionado con el mundo de la empresa, el cambio es permanente (Mariotti et al., 2005).

\section{Poseen una fuerte convicción}

A pesar de las dificultades con las que se pueden encontrar en su labor, los emprendedores siempre creen alcanzar el éxito al final del camino (Staff y Fondas, 1992; Mariotti et al., 2005). Esta convicción les permite navegar a pesar de los obstáculos y problemas y llevar la nave de la empresa al éxito (Kiggundu, 2002).

\section{Espíritu innovador y creatividad}

Los emprendedores promueven la innovación y la creatividad (Marinelli, 1998; Casson, 1982). McGinnis (1987) establece que un emprendedor "cree en la innovación y es creativo, pero con una imaginación pragmática”. 
La competitividad de todos los sectores hace necesarias soluciones y planteamientos creativos para poder obtener el éxito empresarial (Pisani y Patrick, 2002; Kuratko y Montagno, 1989; Drucker, 1985; Stevenson y Jarillo, 1990). Esto es aún más cierto en las nuevas empresas, aquellas que pretenden alcanzar un sitio en un mercado deben innovar constantemente (Ibrahaim y Soufani, 2002; Mariotti et al., 2005).

Schumpeter (1934) ha introducido el concepto de innovación como la base de una persona con actitudes emprendedoras y manifiesta que los emprendedores son quienes innovan en los siguientes aspectos: 1 . Introducción de buenos y mejores servicios/productos; 2 . Nuevos métodos de producción; 3. Manejo de nuevos mercados; y 4. Manejo de una nueva organización para un buen sector de la industria. Schumpeter denominó lo anterior como "la destrucción creativa; se transforman maneras viejas de hacer las cosas".

Shapero (1975) establece que la creatividad es un factor que normalmente se encuentra en todos los empresarios exitosos. Los emprendedores "necesitan expresar su creatividad y a la vez ganarse la vida" (Watson, HogarthScott y Wilson, 1994).

\section{Orientación al mercado y a las oportunidades}

Un rasgo común de los grandes emprendedores es su decidida orientación hacia el mercado, la que les permite identificar oportunidades de negocio atractivas, lo que Caird (1988) denomina "buen olfato para los negocios", sin pensar en el momento inicial de estrategias o recursos necesarios para la posterior gestión (Marinelli, 1998; Moses, 2002). "Los emprendedores son aquellos que siempre buscan cambios, reaccionan ante los mismos y los utilizan como nuevas oportunidades" (Drucker, 1974; Carree y Thurik, 2002 McClelland, 1987; Bird, 1989; Ibrahim y Ellis, 1990; Timmons, 1994; Livinstong, 1971; Greenberger y Sexton, 1988). Moen et al. (2004), afirma que el emprendedor "explota las oportunidades para su ganancia personal a lo largo del tiempo". Schumpeter (1934) establece que "los emprendedores buscan promover el cambio y generan nuevas oportunidades".

\section{Son visionarios y soñadores}

McGinnis (1987) expresa que la visión es la habilidad de integrar las experiencias pasadas en nuevas series que le permiten ser innovador. En tanto que para Hatch et al. (2000) visión es la habilidad de percibir una oportunidad donde otros no pueden.

Los emprendedores saben lo que quieren y saben cómo conseguirlo (Moen et al., 2004). "Invierten la mayor parte de su tiempo mirando hacia adelante" (Marinelli, 1998). Tienen desde el momento inicial una clara visión de su proyecto de empresa y de cómo ésta evolucionará (Rae y Carswell, 2001). En muchas ocasiones, esta visión inicial se irá desarrollando y el emprendedor adaptará su proyecto a nuevos acontecimientos del entorno o a nuevos objetivos corporativos. "El emprendedor construye una visión prácticamente de la nada" (Timmons, 
1994). "Es el soñador que deduce cómo convertir una idea en una realidad aprovechable" (Pinchot, 1987). Puedo agregar que los emprendedores son soñadores, pero con los pies en la tierra. Liderazgo

En palabras de Hisrich (1992), poseen la visión de un líder. Tienen la "habilidad para inspirar a otros" (Kiggundu, 2002). "Motivan e inspiran a los demás para unirse o quedarse con la nueva aventura" (Covey, 1999). "Liderazgo para comunicar, convencer y motivar a su equipo de trabajo, dirigirlo, estimularlo, orientarlo, delegar autoridad y responsabilidad, infundir confianza, fomento de la creatividad, la unión del equipo en torno a su persona y con un sentimiento de misión que se concreta en la propia empresa" (Mariotti et al., 2005). Los emprendedores poseen habilidades de dirección y deseo por el mando" (Kent et al., 1982; Montagno et al., 1986; Begley et al., 1987; Hatch et al., 2000; Rotter, 1966; Ibrahaim y Soufani, 2002).

Creación de redes de apoyo

Los emprendedores siempre se refieren al grupo que les ayudó a producir sus éxitos (Covey, 1999). Son personas con gran disposición y capacidad de cooperación y colaboración con otros (Casson, 1982; McGinnis et al., 1987). "En lugar de individuos iconoclastas, de los capitalistas vaqueros de los sueños norteamericanos, nos encontramos personas enredadas e imbuidas en industrias, con ricas redes de contactos y colegas a quienes podían recurrir para que los ayudaran a construir una empresa. Para la mayoría, el secreto del éxito en su gestión empresarial no parecía radicar solamente en la inspiración individual, sino en reunir decenas de intereses diferentes en un esfuerzo cooperativo. Crear una compañía fue cuestión de conocer a clientes, abastecedores, socios y fuentes de capital" (Mariotti et al., 2005).

Realizando una observación con respecto al listado anterior de características emprendedoras, tal y como lo afirma González Domínguez (2002), "sería utópico pensar que todos los emprendedores reúnen estas cualidades, pero en cierta medida si no las desarrollan o tienen en uno u otro grado, antes o después les pasará la factura".

La Figura 1 sintetiza las principales características que un emprendedor de éxito debe reunir, tomando en consideración el listado anterior.

\section{Un modelo tentativo sobre el comportamiento emprendedor}

González Domínguez (2005) se pregunta: ¿Por qué se crean nuevas empresas? ¿Basta con que un emprendedor tenga una idea para que se decida a crear una empresa? ¿Cualquier persona que tenga una idea es emprendedora y puede ponerla en marcha creando su empresa? En el estudio del emprendedurismo surgen estas interrogantes una y otra vez, y cada teoría intenta darles respuesta desde su propia perspectiva.

El modelo que se presenta en el Diagrama 1, es una aproximación a esta realidad emprendedora. Para desarro- 
Figura 1. Resumen de CEPs del emprendedor de éxito.

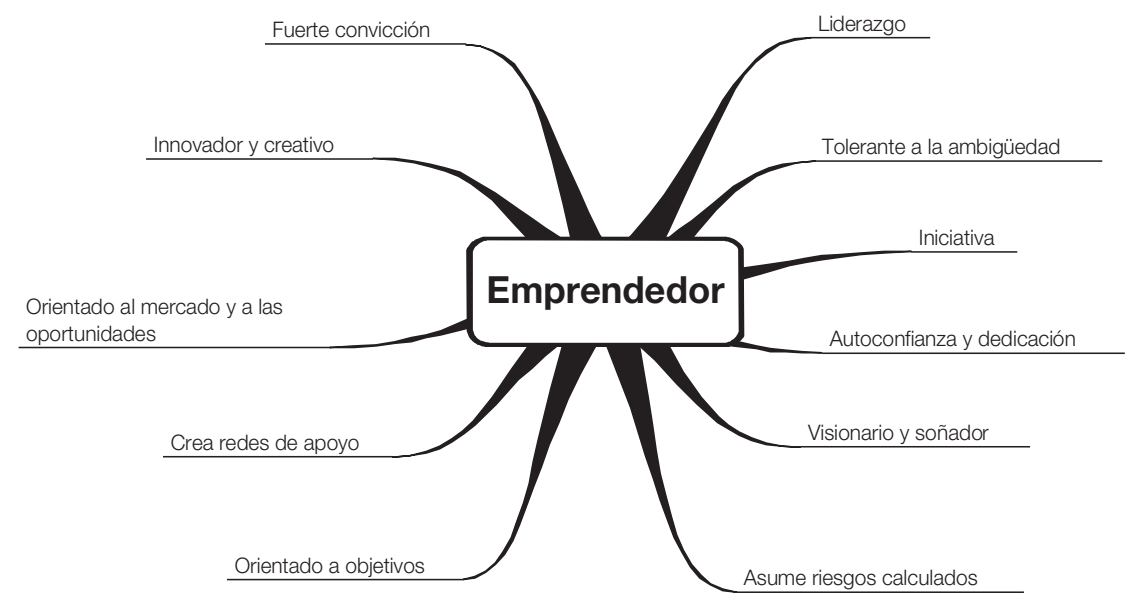

Elaboración: Autor.

llar la actitud emprendedora, en primer lugar, se debe tener una idea de la posterior empresa (Schumpeter, 1934; Carree y Thurik, 2002; Fishbein y Ajzen, 1976; Pinchot, 1985; Ronstadt, 1984), lo que se refleja en el componente cognoscitivo. Tal y como lo afirma Bumm (1988), "sus ideas de negocios reflejan la frescura de la adolescencia, ya que los emprendedores ven al mundo con ojos de juventud". Asimismo debe anhelar fundar su organización, el componente emocional, o como lo llaman algunos autores el "deseo de ser independiente y tener autonomía” (Auken, 1999; Boyd y Gumpert 1983; Montagno y Kuratko, 1986; Begley y Boyd, 1987; McClelland, 1987), y este "deseo dependerá de la valoración que haga de múltiples factores y circunstancias (tranquilidad, aversión al riesgo, necesidad de logro, entre otros)".

El último es el componente de comportamiento, que influye en gran medida; Herron y Robinson (1993) encontraron que el comportamiento y las habilidades del empresario tienen un impacto significativo en el funcionamiento de la empresa. Sin embargo, autores han indicado que la personalidad también tiene un impacto considerable en la decisión de lanzamiento y en el comportamiento (Carter, Gartner, y Reynolds, 1996; Morris, 2003). En tanto que Robinson et al. (1991) argumenta que las actitudes personales son los mejores predictores de las tendencias y características emprendedoras.

Aunado a lo anterior, también existen una serie de factores sociodemográficos que intervienen en la actitud emprendedora desde el impacto que tienen en sus diversos componentes, a saber: género, educación (Johnson, 1994), cultura y valores (Hatch, 2000; Kiggundu, 2002; McClelland, 1961; Dunkelberg y Cooper, 1982; Hornaday y Aboud, 1971; Timmons, 1978), y an- 


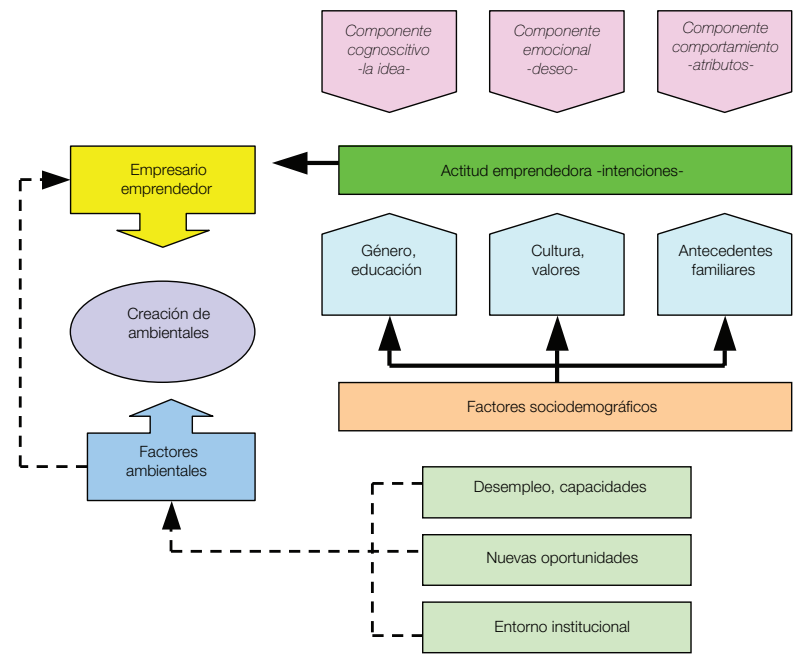

Fuente: González Domínguez (2005).

tecedentes familiares (Shaver y Scout, 1991).

Con relación al género, Brenner (1982) expresa que "si una mujer tiene éxito, ella no cumple con las expectativas sociales sobre su rol femenino y experimenta consecuencias negativas, como la impopularidad y la pérdida de feminidad"; en consecuencia, los hombres son más dominantes y están orientados más al logro que las mujeres, esto explicaría el porqué los varones son más propensos a crear empresa que las mujeres, aunque para otros autores esto es "debido a características psicológicas" (Sexton y Bowman-Upton, 1990). S. Sonfield et al. (2001) encontraron que no existen diferencias significativas de género en cuanto a las situaciones de innovación/riesgo o las estrategias elegidas por los propietarios de las empresas. Las empresarias estaban más influidas por situaciones desagradables y buscaban en la empresa un medio de compatibilizar sus propias necesidades y las de sus hijos (Cromie, 1987).

Los valores y la cultura tienen mucha influencia en el desarrollo de una actitud emprendedora. Ya lo afirma Godley (2001), cuando dice que "parece razonable suponer que la cultura pudo ser de importancia particular cuando viene a explicar variaciones en la fuente de emprendedurismo. El emprendedurismo es una actividad donde se toma mucho riesgo, en contraste a la alternativa de tener un salario fijo en el trabajo. La fuente del emprendedurismo dentro de una economía puede darse no solamente por relación a los beneficios económicos, sino también debido a ciertos valores culturales referentes al riesgo y a la incertidumbre".

Otro aspecto interesante a considerar son los antecedentes familiares, en donde se nota que "muchos emprendedores descienden de familias 
en las que alguno de sus miembros ha sido o es empresario o autoempleado, facilitándose así una cultura y unos valores proclives al emprendedurismo" (González Domínguez, 2005). Morris (1998: 68) plantea que los antecedentes familiares son muy importantes, y que una vida personal con experiencia familiar autoritaria de parte de los progenitores

impulsa al emprendedurismo, en tanto que una familia unida, paternalista, proteccionista, influye en que exista un nivel muy bajo, igual sucede con la educación, experiencias de grupo y experiencias de trabajos anteriores. Esto puede verse en la Figura 2.

Se incluyen también los factores ambientales, que según González Domínguez (2005) son muy diversos y constituyen, uno o varios de ellos, como los desencadenantes de la actividad emprendedora, y son los motivos que en diversas investigaciones los emprendedores emplearon para provocarles y dar el primer paso en la creación de su empresa. Entre estos factores se mencionan los siguientes: pérdida del trabajo, deseo de independencia, oportunismo, ser su propio jefe, aprovechamiento de la experiencia adquirida, una salida ante la falta de alternativas laborales, y aprovechar ayudas institucionales.

La educación influye de manera positiva en los emprendedores (Morris, 1998). Existen registros donde se sugiere que la enseñanza no convencional ayuda a los empresarios a tener éxito; de hecho, los empresarios actuales tienen altos niveles de estudio y probablemente sean graduados de la universidad, y una experiencia educativa creativa y ambigua, eleva el nivel de emprendedurismo (Morris, 1998).

Kolchin et al. (1987), con relación a la educación, expresa que "no se puede predecir de dónde los emprendedores florecerán, se requiere mejor hacer un trabajo cultivando el espíritu emprendedor que está en todos nosotros", lo que Rae y Carswell (2001) denominan "aprender a emprender". Hoy se sugiere que la enseñanza no convencional ayuda a los empresarios a alcanzar el éxito (Tortella y otros, 2010). Silva Duarte (2009) establece que el ambiente educativo es fundamental, dado que el niño y el joven afianzan dos papeles esenciales para el ejercicio de la vida adulta: la sumisión y la autonomía.

Figura 2. Experiencias vividas y emprendedurismo.

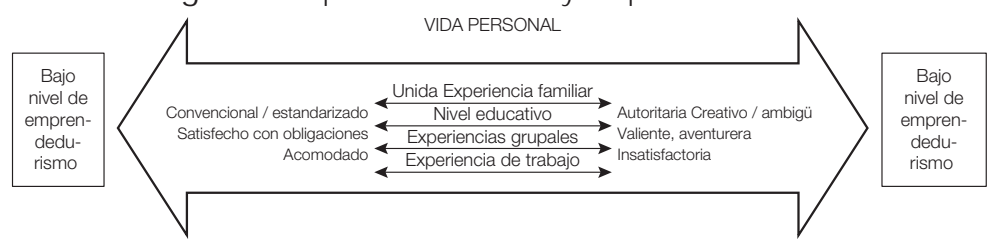

Fuente: Adaptado de Morris (1998, p. 68).

Retos 2(1): 2011.

(C) 20II, Universidad Politécnica Salesiana del Ecuador 


\section{Impacto de la educación "emprendedora"}

Comencemos este apartado con una pregunta: ¿impacta la educación en el desarrollo del espíritu emprendedor? El premio Nobel de Economía, T. W. Shcultz (1985) afirma que la educación es una de las vías que influyen positivamente en el surgimiento de nuevos empresarios, personas capaces de tratar con el desequilibrio. Se deduce que a mayor nivel educativo surgirán más personas dispuestas a crear nuevas empresas.

M. Casson (1991) señala que el sistema educativo contribuye inicialmente al desarrollo de las capacidades requeridas por el empresario. En este sentido un nivel de educación superior resulta ser una ventaja frente a quienes no lo alcanzan. En el estudio de Kourilsky (1998), donde los niños simulan ser empresarios, la habilidad académica es una de las variables que mejor predice el éxito empresarial.

Pero, por otro lado, el mismo Casson señala que para un empresario la enseñanza superior no le es imprescindible. Ser empresario no significa ser profesionalmente competente en todas las tareas empresariales (producción, administración, gerencia, comercialización, etcétera). Su tarea más bien consiste en conseguir para la empresa a profesionales competentes, delegar en ellos funciones específicas, dirigirles hacia el logro de los objetivos, motivarles, etcétera.

Alicia Castillo (1999) refiere que "la enseñanza de emprendimiento, debe fundamentarse en la Acción
Basada en la Teoría (en inglés TBA). Todos los trabajos anteriores se refieren a teorías y modelos desarrollados para ser aplicados en capacitación, bien sea a estudiantes de cursos de pre y posgrado como a ejecutivos y emprendedores. Los educadores de emprendimiento deben relacionar teoría con práctica”. En este sentido, será necesario entonces que los facilitadores desarrollen actividades prácticas y vivenciales para que el entorno de aprendizaje sea favorable al estudiante, principalmente para el desarrollo de sus habilidades emprendedoras.

\section{Algunos resultados prácticos} Factor educación y perfil emprendedor Para el desarrollo de esta investigación se visitó a 1.059 emprendedores en todo el país, los principales resultados en la temática de la educación fueron los siguientes:

Con respecto al nivel de educación que presentan los emprendedores en El Salvador (ver Tabla 1), un 41,8\% del total tiene estudios de Bachillerato $(26,6 \%$ mujeres y $15,2 \%$ hombres). El grupo poblacional que le sigue es el que posee un grado universitario con $19,5 \%(9,7 \%$ mujeres y $9,8 \%$ hombres). Un 11,6\% de los emprendedores dijo poseer estudios de hasta noveno grado (6,7\% mujeres y $4,9 \%$ hombres). Otro $10 \%$ del total dijo haber ido a la Universidad pero que no había culminado sus estudios (4,9\% mujeres y $5,1 \%$ hombres). También hay que señalar que un $10,1 \%$ de los emprendedores dijo no poseer ningún tipo de estudio (6,6\% mujeres y $3,5 \%$ hombres). 
Debe aclararse que en El Salvador, la educación se divide en:

- Primaria (primero a sexto grado), seis años si no se repite grado;

- Secundaria(séptimo a noveno grado), tres años si no se repite grado;
- Bachillerato (General de dos años y Técnico de tres años);

- Universitario (cinco años para una carrera de grado) (Para acceder a la universidad se requiere ser bachiller).

Tabla 1. Nivel de estudio de los emprendedores (por género).

\begin{tabular}{|c|c|c|c|c|c|c|}
\hline \multirow[t]{2}{*}{ Nivel de estudios } & \multicolumn{4}{|c|}{ Género } & \multirow[t]{2}{*}{ Total } & \multirow[t]{2}{*}{$\%$} \\
\hline & Femenino & $\%$ & Masculino & $\%$ & & \\
\hline Ninguno & 70 & $6.60 \%$ & 37 & $3.50 \%$ & 107 & $10.10 \%$ \\
\hline Hasta novena grada & 71 & $6.70 \%$ & 52 & $4.90 \%$ & 123 & $11.60 \%$ \\
\hline Bachillerato & 282 & $26.60 \%$ & 161 & $15.20 \%$ & 443 & $41.80 \%$ \\
\hline Diplomados/Técnicos & 3 & $0,30 \%$ & 1 & $0.10 \%$ & 4 & $0.40 \%$ \\
\hline Universitarios inconclusos & $\$ 2$ & $4.90 \%$ & 54 & $5.10 \%$ & 106 & $10.00 \%$ \\
\hline Titulo Universitario & 103 & $9.70 \%$ & 104 & $9.80 \%$ & 207 & $19.50 \%$ \\
\hline Doctorado & 2 & $0.20 \%$ & 1 & $0.10 \%$ & 3 & $0.30 \%$ \\
\hline Maestría & 27 & $2.50 \%$ & 37 & $3.50 \%$ & 64 & $6.00 \%$ \\
\hline Otros & 2 & $0.20 \%$ & 0 & $0.00 \%$ & 2 & $0.20 \%$ \\
\hline TOTAL & 612 & $57,80 \%$ & 447 & $42.208 \%$ & 1059 & $100.00 \%$ \\
\hline
\end{tabular}

Elaboración: Autor.

\section{Comparación de la educación convencional con la educación emprendedora}

A continuación presento una tabla comparativa entre desarrollar una "educación convencional" y la aplicación de una "educación emprendedora" en el salón de clases:

Tabla 2. Educación Convencional vs. Educación Emprendedora.

\section{EDUCACIÓN CONVENCIONAL}

El contenido visto como meta.

Conducido y dominado por el instructor.

El instructor repasa el conocimiento.

Adquisición de informaciones "correctas".

Currículo y sesiones fuertemente programadas.

Objetivos de la enseñanza impuestos.

\section{EDUCACIÓN EMPRENDEDORA}

Énfasis en el proceso; aprender a aprender. Apropiación del aprendizaje por el participante.

El instructor como facilitador y educando: los participantes generan el conocimiento.

Lo que se sabe puede cambiar.

Sesiones flexibles y orientadas a necesidades.

Objetivos del aprendizaje negociados. 


\begin{tabular}{l|l} 
Centrada en la transmisión de conocimientos. & $\begin{array}{l}\text { Centrada en la formación de competencias: } \\
\text { saber ser, saber hacer, saber estar, saber } \\
\text { convivir. }\end{array}$ \\
$\begin{array}{l}\text { Rechazo el desarrollo de conjeturas y pensa- } \\
\text { miento divergente. }\end{array}$ & $\begin{array}{l}\text { Coyunturas y pensamiento divergentes; } \\
\text { vistos como parte del proceso creativo. } \\
\text { Envolvimiento de todo el cerebro, aumento } \\
\text { de la racionalidad del cerebro izquierdo a tra- } \\
\text { vés de las estrategias holísticas, no lineales, } \\
\text { intuitivas; énfasis en la confluencia y fusión de } \\
\text { los dos procesos. }\end{array}$ \\
$\begin{array}{l}\text { Énfasis en el pensamiento analítico y lineal: } \\
\text { parte izquierda del cerebro. }\end{array}$ & $\begin{array}{l}\text { Aliento a la influencia de la comunidad. } \\
\text { Experiencia interior es contexto para el } \\
\text { aprendizaje; sentimientos incorporados a la } \\
\text { Resistencia a la influencia de la comunidad }\end{array}$ \\
$\begin{array}{l}\text { Énfasis en el mundo exterior; la experiencia } \\
\text { interior es considerada impropia al ambiente } \\
\text { escolar }\end{array}$ & $\begin{array}{l}\text { Educación encarada como necesidad social } \\
\text { durante cierto período de tiempo, para afir- } \\
\text { mar habilidades mínimas para un determina- } \\
\text { do papel. }\end{array}$ \\
$\begin{array}{l}\text { Errores no aceptados. } \\
\text { la vida, relacionado apenas tangencialmente } \\
\text { con la escuela. }\end{array}$ \\
$\begin{array}{ll}\text { El conocimiento es el vínculo entre el alumno } \\
\text { y el profesor, elemento de fundamental } \\
\text { importancia. }\end{array}$ & $\begin{array}{l}\text { Errores como fuente de conocimiento. } \\
\text { La estrecha relación académica entre } \\
\text { profesores y alumnos es de fundamental } \\
\text { importancia. }\end{array}$ \\
\hline
\end{tabular}

Fuente: Programa de Educación Emprendedora, Aldeas Infantiles SOS, Bolivia.

En la actualidad, las instituciones educativas se encuentran trabajando hacia una educación que desarrolle competencias en el educando, que fortalezca sus habilidades y destrezas, y desde este punto de vista hemos pasado de una educación tradicional, centrada en el docente, hacia una educación centrada en el estudiante, en donde las metodologías se enfocan en el "saber-hacer" y los conocimientos son contrastados en la realidad por cada estudiante.

Pittaway y Cope (2007) distinguen entre la formación sobre el emprendimiento (about entrepreneurship), de tipo conceptual; la formación para el emprendimiento (for entrepreneurship), vocacional y centrada en la adquisición de habilidades técnicas, y la formación a través del emprendimiento (practice in or through), y se refiere a la forma como se aprende a ser empresario; "el punto central en el aprendizaje es llegar a ser practicante, no aprender sobre la práctica".

Klandt y Volkmann (2006) enuncian tres principios aparentemente evidentes (self evident) sobre el aprendizaje del emprendimiento:

- Puede ser aprendido: no es exclusivamente una habilidad innata.

- Puede ser enseñado: no se aprende solo con la experiencia.

- Puede adquirirse a través de cursos universitarios.

\section{¿Qué retos enfrenta el docente?}

En el nuevo rol que el docente ha de desempeñar, "se debe abandonar el 
papel de poseedor del saber para convertirse en un promotor de la generación y construcción del conocimiento" (Porro, 2008). Surgen de esta manera competencias que deben ser cultivadas por todo educador, tales como:

- Plantear una problemática en particular y promover el abordaje de esta por parte del grupo, desde diversas ópticas.

- Administrar las intervenciones grupales y en plenaria.

- Registrar y subrayar los conceptos surgidos por los miembros del grupo, rescatando los aportes más destacados.

- Promover la participación de todos, que ningún estudiante se quede sin participar.

- Alentar y hacer descubrir la valía personal que cada alumno tiene, desde sus propias particularidades.

- Orientar y reflexionar con el grupo basado en su mayor experiencia de vida, manteniendo el eje de la discusión, pero convirtiendo al estudiante en el protagonista y responsable de su propio aprendizaje.

El educador en sí mismo es capaz de transmitir la fuerza y pasión del emprendimiento y para ello se debe "educar en una actitud emprendedora" (Díaz Abajo, 2007), en el diseño y en el desarrollo de su clase.

Para Alicia Castillo (1999), las nuevas tendencias en formación emprendedora implican el uso combinado de teoría para explicar el éxito o fracaso de un caso. Se espera que la enseñanza del emprendimiento genere un cambio de actitud, por lo tanto, el profesor es un actor irremplazable en la generación de una mentalidad diferente. Dar clases de emprendimiento por lo tanto, representa un desafío que enriquece a ambas partes. El contrato entre profesores y estudiantes es de doble compromiso. Por un lado, se requiere el esfuerzo especial del profesor para:

1. Prepararse cuidadosa y completamente para cada clase.

2. Manifestar preocupación y devoción real a los estudiantes, y aún a aquellos que han dejado de serlo, en la clase y en la oficina.

3. Motivación para hacer que el curso sea una experiencia de desarrollo significativa para los estudiantes.

Por otro lado, mientras más dé el profesor, más podrá exigir, y en el proceso, las clases podrán ir mejorando continuamente.

El enlace entre la teoría y la práctica a su vez, es explicada por Bill Bygrave (referido por Alicia Castillo, 1999), Director del Centro de Emprendimiento de Babson College. Hay dos maneras de arruinar un curso de emprendimiento:

- La primera es basándola totalmente en la aplicación práctica y el análisis de casos (un curso que solo tenga aplicaciones prácticas y casos no permite que los estudiantes actúen en base a sus decisiones).

- La segunda es hacerlo totalmente teórico (un curso de pura teoría puede considerarse como un botadero árido, donde solo los estudiantes más curiosos intelectualmente progresen. La mayoría de los estudiantes se aburren y no internalizan el conocimiento). 


\section{Nueva perspectiva del rol de la universidad en la educación emprendedora}

El autor Per Blenker (2006) propone el siguiente esquema, en el cual la Universidad juega un rol sumamente importante en el desarrollo del espíritu emprendedor.

Figura 3. El rol de la Universidad en la formación emprendedora.

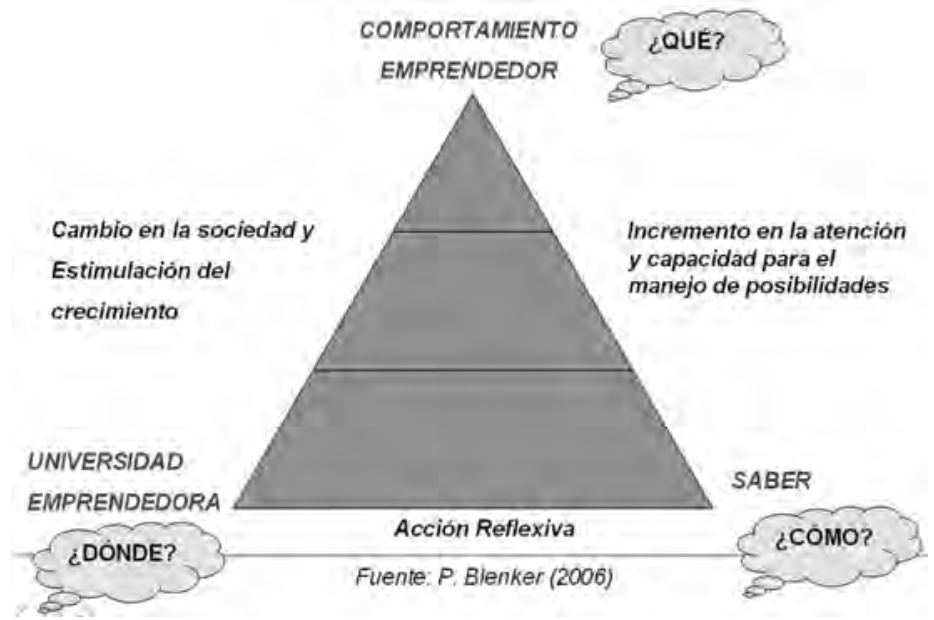

Fuente: P. Blenker (2006).

Los tres ejes de la pirámide de Blenker (ver Figura 3) son: el saber, la universidad emprendedora y el comportamiento emprendedor. La 'universidad emprendedora' y el 'saber' fomentan y desarrollan la 'acción reflexiva'; el 'saber' estimula el incremento en la atención y la capacidad para que la persona pueda desempeñarse en distintos ambientes, estimulando el 'comporta- miento emprendedor'. Y la 'universidad emprendedora' a partir del 'comportamiento emprendedor', generan un cambio en la sociedad y estimulación del crecimiento. La 'universidad emprendedora' responde al 'contexto'; el Saber responde a la "pedagogía", y el 'comportamiento emprendedor' a la 'didáctica', según Blenker.

Figura 4. Evolución educativa en forma jerárquica.

\begin{tabular}{|c|c|c|c|}
\hline ¿Qué? & ¿Qué conocer? & ¿Cómo conocer? & ¿Cómo hacer? \\
\hline Atención & Interés & Entendimiento & Acción \\
\hline \multicolumn{2}{|c|}{ Partiendo de la base e incrementando el conocimiento y las habilidades } \\
\hline
\end{tabular}

Fuente: P. Blenker (2006).

Retos 2(I): 20II.

C) 20II, Universidad Politécnica Salesiana del Ecuador 
Educación y competencias emprendedoras expresadas en efecto jerárquico

El esquema anterior (Figura 4), propuesto por Blenker, evalúa el desarrollo en los aprendizajes que un estudiante tiene, desde el llamar su atención respondiendo a la pregunta ¿qué?, despertar su interés con ¿qué conocer?, motivar su entendimiento y razonamiento con ¿cómo conocer?, hasta llegar a la práctica de la acción misma, el aprende haciendo a través del ¿Cómo hacer? Este es el proceso evolutivo que se desliza desde la enseñanza "centrada en la formación" hasta el "desarrollo de competencias". Lo ideal es que en cualquier asignatura que impartamos debemos tratar de llegar a la acción, a la práctica, al "aprender haciendo", para desarrollar en el estudiante habilidades y destrezas que le serán de gran utilidad para su desenvolvimiento en la vida profesional.

Timmons ha defendido en múltiples oportunidades que los emprendedores no nacen, sino que se forman, $y$ que independientemente de la formación del individuo, en cualquier etapa de su vida puede adquirir la motivación para ser emprendedor (Castillo, 1999).

Aliaga y Schalk (2010) proponen que para la consecución exitosa de una política, diseño curricular o institucional debieran considerarse los siguientes aspectos:

- Adopción del enfoque de competencias de empleabilidad como combinación de capacidades fundamentales de orden cognitivo superior y genéricas.
- Existencia de estándares exigentes para la formación de dichas competencias.

- Enseñanza basada en los lugares de trabajo.

- Sólidos vínculos entre empleadores y centros de estudios.

\section{Modelo de D. Kolb}

D. Kolb (2001) incluye el concepto de estilos de aprendizaje dentro de su modelo de aprendizaje por experiencia y lo describe como "algunas capacidades de aprender que se destacan por encima de otras como resultado del aparato hereditario de las experiencias vitales propias y de las exigencias del medio ambiente actual (...) Llegamos a resolver de manera característica los conflictos entre el ser activo y reflexivo y entre el ser inmediato y analítico. Algunas personas desarrollan mentes que sobresalen en la conversión de hechos dispares en teorías coherentes y, sin embargo, estas mismas personas son incapaces de deducir hipótesis a partir de su teoría, o no se interesan por hacerlo; otras personas son genios lógicos, pero encuentran imposible sumergirse en una experiencia y entregarse a ella".

Kolb identificó dos dimensiones principales del aprendizaje: la percepción y el procesamiento. Decía que el aprendizaje es el resultado de la forma como las personas perciben y luego procesan lo que han percibido (ver Figura 5).

A partir de ello, describió dos tipos opuestos de percepción: 1. Las personas que perciben a través de la experiencia concreta, y 2. Las personas que perci- 
ben a través de la conceptualización abstracta (y generalizaciones).

A medida que iba explorando las diferencias en el procesamiento, Kolb también encontró ejemplos de ambos extremos:

- Algunas personas procesan a través de la experimentación activa (la puesta en práctica de las implicaciones de los conceptos en situaciones nuevas).

- Otras a través de la observación reflexiva.

La yuxtaposición de las dos formas de percibir y las dos formas de procesar es lo que llevó a Kolb a describir un modelo de cuatro cuadrantes para explicar los estilos de aprendizaje, a saber:

- Involucrarse enteramente y sin prejuicios a las situaciones que se le presenten.
- Lograr reflexionar acerca de esas experiencias y percibirlas desde varias aproximaciones.

- Generar conceptos e integrar sus observaciones en teorías lógicamente sólidas.

- Ser capaz de utilizar eses teorías para tomar decisiones y solucionar problemas.

\section{Red Conceptual de Garavan}

El modelo de Kolb (1984), aplicándolo a la educación emprendedora, y partiendo que todos aprendemos de manera distinta, fue retomado por Garavan (1994) quién desarrolló cada uno de los cuadrantes y propuso las técnicas que un docente podría utilizar de acuerdo a cada perfil de estudiante, lo que se muestra a continuación en la Figura 6.

Figura 5. Representación gráfica del Modelo de D. Kolb.

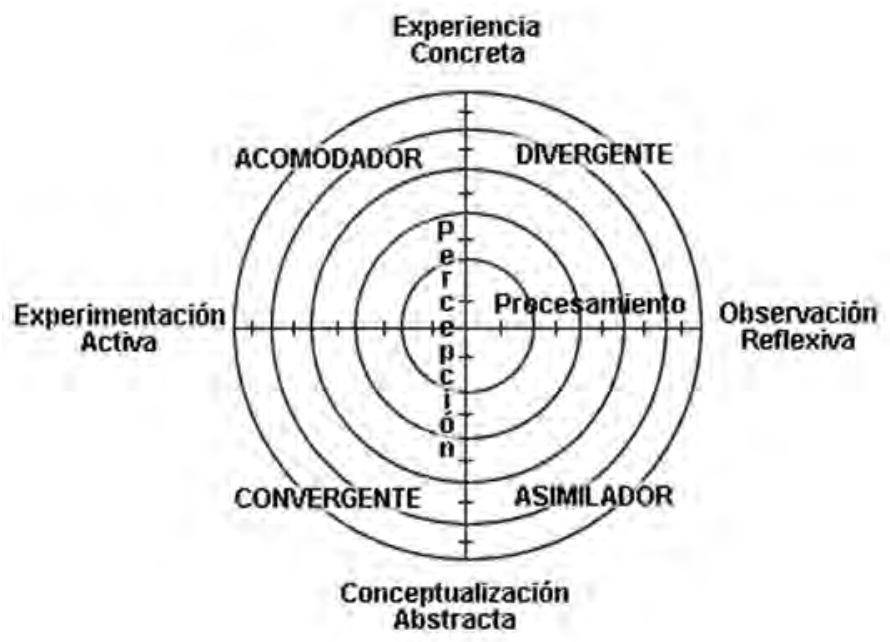

Fuente: Kolb (1948) citado por Lozano (2000: 71).

Retos 2(I): 201I

(C) 20II, Universidad Politécnica Salesiana del Ecuador 
Educación emprendedora en la universidad: Educando para el futuro

Figura 6. Red conceptual de estilos de aprendizaje y técnicas pedagógicas. EXPERIENCIA CONCRETA

\begin{tabular}{|c|c|}
\hline $\begin{array}{l}\text { III. APLICACION ACTIVA } \\
\text { Cambios en las habilidades y actitudes } \\
\text { Juego de Roles } \\
\text { Simulaciones de negocios } \\
\text { Discusiones } \\
\text { Grupos T } \\
\text { Diario de aprendizaje } \\
\text { Proyectos de campo } \\
\text { Gestion de Grupos de Aprendizaje } \\
\text { Tutorie y consejeria }\end{array}$ & $\begin{array}{l}\text { II. APLICACION REFLEXIVA } \\
\text { Cambios en la aplicación } \\
\text { Motivación } \\
\text { Aplicación des la Iectura y discusion limitadas } \\
\text { Casos } \\
\text { Juego de roles } \\
\text { Examenes orlentados a problemas } \\
\text { instrucaón programada (en habilidades) }\end{array}$ \\
\hline EXPERIMENTACION ACTIVA & OBSERVACION REFLEXIVA \\
\hline $\begin{array}{l}\text { IV. ACTIVO - TEORICO } \\
\text { Cambios en la comprension } \\
\text { Grupos centrados en el aprendizaje } \\
\text { Discusion argumentativa } \\
\text { Experimentacion / Investigación } \\
\text { Lecturas sugendas } \\
\text { Anélisis de documentos } \\
\text { Talleres } \\
\text { Seguimiento } \\
\text { Entrenamiento }\end{array}$ & $\begin{array}{l}\text { 1. RELEXIVO-TEORICO } \\
\text { Cambios en el conocimiento } \\
\text { Lecturas teóricas. } \\
\text { Lecturas requeridas } \\
\text { Folletos } \\
\text { Instrucción programeda (en conceptos) } \\
\text { Documentos téoricos } \\
\text { Exámenes orientados a contenidos }\end{array}$ \\
\hline
\end{tabular}

CONCEPTUALIZACION ABSTRACTA

Fuente: Garavan (1994).

El esquema anterior (Figura 6) es muy rico en información, que todo educador puede aprovechar para desarrollar el máximo de aprendizaje en cada uno de sus estudiantes, potenciando el espíritu emprendedor de cada uno de ellos.

A partir de lo anterior, hacemos eco de la reflexión de Arias y Castillo (2011) cuando afirman que: "el emprendimiento es una capacidad humana que puede aprenderse, desarrollarse y mejorarse de manera continua a través de procesos de formación integrales; y para ello, la teoría es esencial para su aplicación con sentido en la práctica, que debe orientarse al desarrollo de competencias que le permitan al individuo adquirir una visión empresarial y tomar decisiones responsables para su proyecto de vida" (las cursivas son mías).

\section{A modo de conclusión}

Con la evidencia recabada, podemos concluir que nos encontramos ante un cambio de paradigma en el campo formativo-académico, y que lograr una cultura emprendedora requiere de:

- Un enfoque amplio y adecuado; en donde el estudiante sea partícipe de su propio aprendizaje, que deje el papel de actor y se convierta en autor, que el docente sea un guía para la construcción del conocimiento y desarrollo de competencias, acortando de esta manera la distancia entre aprendizaje y experiencia, favoreciendo una permanente vinculación con organizaciones productivas.

- Un contacto cercano entre las empresas, los emprendedores y sus historias; ya que la vinculación universidad-empresa representa una 
oportunidad para que los educandos adquieran experiencia en la práctica, conozcan del camino recorrido por otros emprendedores, sus limitaciones y problemas, y como lograron superar dichos desafíos.

- Las metodologías empleadas en el salón de clases deben ser participativas, en donde los estudiantes "aprendan haciendo", realicen ejercicios de simulación empresarial en situaciones hipotéticas, y donde puedan desarrollar poco a poco su potencial emprendedor.

- La Universidad dispone de una gran oportunidad: integrar una formación que promueva la empleabilidad temprana y el emprendimiento $\left(E^{2}\right)$ desde el primer día de formación de nuestros educandos, de tal manera que un estudiante egresado de la UDB, al finalizar su carrera se pregunte ¿Quiero ser empleado o empleador? Y tenga presente ambos caminos como una opción de vida.

\section{Notas}

[1] Esta es la palabra aceptada en el Diccionario de la Real Academia de la Lengua Española (2011), pero personalmente prefiero "emprendedurismo", que para nuestros países latinoamericanos es lo más usual.

[2] Lo plantean de ésta manera autores como McClelland, 1961; Dunkelberg y Cooper, 1982; Hornaday y Aboud, 1971; Timmons, 1978.

\section{Referencias bibliográficas}

Acs, Z.; Arenius, P. y M. Hay, Minniti Global entrepreneurship Monitor 2004 Executive Report. Babson College and London Business School, 2005.

Aliaga, Claudia; Schalk, Ana, E²: Empleabilidad temprana y emprendimiento. Dos grandes desafíos en la formación superior en Chile. Revista Calidad de la Educación, vol. 33., 2010.
Arias, A. et al., "La educación para el emprendimiento y empresarismo virtual: potenacialidades", Revista Virtual Universidad Católica del Norte, No. 32, Colombia, 2011.

Begley, T. M. et al., Psychological Characteristics Associated whit Performance in Entrepreneurial Firms and Smaller Businesses, Journal of Business Venturing, p. 79-91, 1987.

Blenker, P., Entrepreneurship Education. The New Challenge Facing the Universities, University of Aarhus, Dinamarca, 2006.

Block, Z. et al. Entrepreneurship Education Research: Experience and Challenge in The State of the Art of Entrepreneurship, ed. Donald L. Sexton and John D. Kasarda (Boston, MA: PWS-Kent), p. 17-42, 1992.

Brockhaus, R.H. et al., The Psychology of the Entrepreneur, The Art and Science of Entrepreneurship, Ballinger, Cambridge, M A., 1986.

Carree, M. A. et al., The Impact of Entrepreneurship on Economic Growth, Faculty of Economics and Business Administration, University of Maastricht, Chapter prepared for the International Handbook of Entrepreneurship Research, July, 2002.

Castillo, Alicia, Estado del arte de la enseñanza del emprendimiento. Proyecto "Emprendedores como generadores de riqueza y desarrollo regional", First Public Inc., Chile, INTEC-Chile, 1999.

Casson, M., The Entrepreneur: an Economic Theory, Martin Robertson, Oxford, 1982.

Cromie, S., Motations of aspiring male and female entrepreneurs, Journal of Occupational Behavior, n. 8, pp. 87-113, 1987.

Cunningham, J. B. et al. Defining Entrepreneurship, Journal of Small Business Management (January): 45-61, 1991.

De Dios Uriarte, Juan, Características psicológicas diferenciales de los jóvenes emprendedores, Tesis Doctoral presentada en la Universidad del País Vasco, España.

Díaz Abajo, A. Educar para emprender, Revista Andalucía Educativa No. 59, España. Web site: www.juntadeandalucia.es, 2007.

Dunkelberg, W. C. et al. Entrepreneurial typologies. en K. H. Vesper (Ed.), Frontiers of entrepreneurship research, 1982.

Garavan, T. N., \& O'Cinneide, B. Entrepreneurship education and training programmes: A review and evaluation. Journal of European Industrial Training, 18(8), 3-12, 1994.

González Domínguez, F. J., Creación de empresas. Guía para el desarrollo de iniciativas empresariales, Madrid, Ed. Pirámide, p. 56$59,2005$. 
Gutiérrez Montoya, Guillermo, El comportamiento emprendedor en El Salvador, Tesis de Doctorado en Ciencias Empresariales, dirigida por el Dr. Ángel Cervera Paz y el Dr. Francisco Rodríguez, España, Universidad de Cádiz, 1999.

Harding, R., Global Entrepreneur ship Monitor: United Kingdom 2002. London, UK, London Business School, 2002.

Herron, L.; Sapienza, H. J., y D. Smith Cook, Entrepreneurship Theory from an Interdisciplinary Perspective, Entrepreneurship Theory and Practice, p. 5-12, 1992.

Herron, L. et al., A structural model of the effects of entrepreneurial characteristics on venture performance, Journal of Business Venturing, vol. 8, pp.281-294. 1993.

Hisrich, R. D., The Entrepreneuer in Northern Ireland: Characteristics, Problems, and Recommendations for the future, Journal of Small Business Management; July, 1988.

Hornaday, J. A. et al. (1971): Characteristics of successful entrepreneurs, Personnel Psychology, p. 141-153, 2011.

Ibrahaim, A. B. et al. Entrepreneurship education and training in Canada: A critical assessment, Education $\mathcal{B}$ Training, London, Tomo 44, No. 8/9; p. 421, 2002.

Kantis, H. et al. Desarrollo Emprendedor: América Latina y la experiencia internacional, BID, USA, 2004.

Klandt, Heinz; Volkmann, Christine, Development and Prospects of Academic Entrepreneurship Education in Germany. Higher Education in Europe, vol. 31, No. 2, pp. 195 208, 2006.

Kolb, A., \& D. A. Kolb, Learning styles, en Forest, J. Eु Kinser, K. (Eds.), Encyclopedia of Higher Education in the United States, ABCCLIO Publishers, 2001.

Kolchin, M. G., y T. J. Hyclak, The case of the Traditional Intrapreneur, S. A. M. Advanced Management Journal, USA, 1987.

Kourilsky, M. L., \& W. B. Walstad, Entrepreneurship and female youth: Knowledge, attitudes, gender differences, and educational practices. Journal of Business Venturing, 13(1), 77-88, 1998.

Kuratko, D. F., New Venture Creation: A Laboratory Course for Entrepreneurship Education, Journal of Business Education (March), p. 248-50, 1989.
López, I. (2005): El emprendizaje Social: motor de desarrollo y cohesión social, Ponencia presentada en la Universidad Autónoma de Madrid, España.

Lozano, A., Estilos de aprendizaje y enseñanza. Un panorama de la estilística educativa, ITESM, Universidad Virtual-ILCE, México, Ed. Trillas, 2000.

Martínez Arias, R., El análisis multivariante en la investigación científica, Cuadernos de Estadística, Madrid, Editorial La Muralla, Hespérides, 144 pp. 1999.

Maslow, A. H., "A theory of human motivation", Psychological Review, 50, 370-396, 1943.

Moreno Rodríguez, J. E., Hacia una pedagogía emprendedora en Educación Superior, Colombia, Universidad Metropolitana, Colombia, 2008.

Morris, M. H., Entrepreneurship as experienced by the entrepreneur, Journal of Developmental Entrepreneurship; Apr; 8, 1, 2003.

Pinchot, G. III, Intrapreneuring, Harper $\mathcal{E}$ Row, Publishers, New York, USA, 1985.

Pittaway, Luke, y Jason Cope, Stimulating Entrepreneurial Learning. Integrating Experiential and Collaborative Approaches to Learning. Management Learning, vol. 38, No. 2, pp. 211-233, 2007.

Porro, Silvia, Investigación-Acción. Una experiencia de docentes de primaria y secundaria en una escuela Argentina, Buenos Aires, Universidad Nacional de Quilmes, 2008.

Sánchez, José C. Entrepreneurship: introduction, Psicothema, Universidad de Salamanca, vol. 23, No. 3, pp. 424-426, 2011.

Schumpeter, J., Change and the Entrepreneur, en Essays of J. A. Schumpeter, ed. Richard V. Clemence, Reading, MA, Addison-Wesley, 255, 1951.

Silva Duarte, J. E., Emprendedor. Crear su propia empresa, México, Ed. Alfaomega, 2009.

Sonfield, Matthew; Lussier, Robert; Corman, Joel; McKinney, Mark Gneder, Comparisons in Strategic Decision-Making: An Empirical Analysis of the Entrepreneurial Strategy Matrix, Journal of Small Business Management, vol. 39, n. 2, pp. 165-173. 2001.

Tortella, G.; Quiroga, G. y I. Moral, "Entrepreneurship: a comparative approach", en The determinants of entrepreneurship. Leadership, culture, institutions, edited by J. L. GarcíaRuíz and P. A. Toninelli, 2010.

Varela, R. Innovación empresarial. ICESI. Colombia, 1998.

\section{Envío 13/septiembre/2011- Aceptación 04/noviembre/2011}

Retos 2(1): 2011.

(C) 201 I, Universidad Politécnica Salesiana del Ecuador 